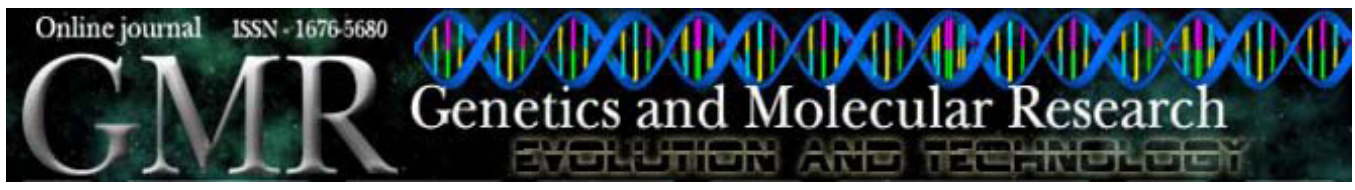

\title{
Expression of GFP can influence aging and climbing ability in Drosophila
}

\author{
R.M.S. Mawhinney and B.E. Staveley \\ Department of Biology, Memorial University of Newfoundland, \\ St. John's, Newfoundland and Labrador, Canada \\ Corresponding author: B.E. Staveley \\ E-mail: bestave@mun.ca
}

Genet. Mol. Res. 10 (1): 494-505 (2011)

Received August 27, 2010

Accepted January 8, 2011

Published March 22, 2011

DOI 10.4238/vol10-1gmr1023

\begin{abstract}
Green fluorescent protein $(G F P)$ is widely used as a reporter transgene in a variety of organisms. Some of the advantages of using GFP include non-invasive visualization of biological events and/or tissues in live specimens and its benign nature. When GFP is expressed throughout the organism, in neurons and eyes, lifespan and climbing ability of flies are significantly decreased compared to similar crosses with a lacZ reporter. Also, GFP expression can have subtle effects on eye morphology, with neural and ubiquitous expression. Since $G A L 4 / U A S$ expression of GFP can influence aging and climbing ability in the Drosophila system of directed gene expression, we found that the latter of these advantages, namely its harmless, non-toxic nature, can be conditional, depending upon the mode of expression and the biological endpoint. We suggest that caution should be used when using GFP to visualize cellular events, especially in long-term assays.
\end{abstract}

Key words: Drosophila melanogaster; Green fluorescent protein (GFP); Longevity; Climbing 


\section{INTRODUCTION}

The green fluorescent protein (GFP) gene, originally isolated from the jellyfish Aequorea victoria, has been a vital tool in biological research, particularly for genetic screening and analyses of gene expression in Drosophila melanogaster. Initial studies used GFP included in P-element constructs, to facilitate localization of proteins and to visualize cell events, such as movement of ribonucleoprotein particles in Drosophila embryos (reviewed by Wang and Hazelrigg, 1994 and Misteli and Spector, 1997). Another gene used in P-element fusions to visualize cell and genomic components is lacZ, a gene from Escherichia coli, which encodes B-galactosidase (O'Kane and Gehring, 1987). Creation of both UAS-GFP (Yeh et al., 1995; Dickson, 1996) and UAS-lacZ reporter lines (Manseau et al., 1997) presented novel ways of investigating gene expression in a variety of animals by using the GAL4/UAS system to direct expression in specific cell or tissue types (reviewed by Chalfie et al., 1994; Rosochacki and Matejczyk, 2002, and Zhan and Gong, 2010). These transgenes have been used as controls to examine effects of gene expression.

Advantages of using the GAL4/UAS system with the GFP reporter include being able to work with living cells with little or no need for sample preparation (reviewed by Misteli and Spector, 1997 and Duffy, 2002). While either GFP or lacZ can be chosen as the reporter gene in certain assays, GFP allows researchers to visualize expression patterns non-invasively and continuously in live specimens, unlike when lacZ/X-Gal staining is used (Yeh et al., 1995; Phelps and Brand, 1998). It is believed that both GFP and lacZ expressions are non-toxic and do not influence endogenous gene expression at any stage during fly development (Bier et al., 1989; Chalfie et al., 1994). Despite this, some studies have suggested that GFP affects cell biology. Cells with GFP-containing constructs that localize GFP in the nucleus tend to be more damaged, possibly due to free-radical generation and damage to DNA (Misteli and Spector, 1997). In analysis of zebrafish development, expression of GFP is delayed and a longer recovery period is required before target genes are activated (Zhan and Gong, 2010). The subtle consequences of GFP expression are of considerable interest to a wide range of biologists.

In addition to these effects of GFP, GAL4 has been shown to affect cell biology in flies. Effects on ommatidia development have been observed and it was established that these altered phenotypes are caused by cell death in the eye (Freeman, 1996; Kramer and Staveley, 2003). In addition, elav-GAL4 reduces longevity (Todd AM and Staveley BE, unpublished results). Examination of transgenes used for driving reporter expression is another route for research.

We find that GFP expression promoted by select GAL4 transgenes can have adverse effects upon morphology and behavior in flies, in particular lifespan and climbing ability, when compared to similar population of flies expressing the lac $Z$ transgene.

\section{MATERIAL AND METHODS}

\section{Fly stocks and crosses}

All Drosophila stocks were obtained from the Bloomington Drosophila Stock Centre (University of Indiana, Bloomington). The two responder fly lines used were $w$; UAS-lac $Z^{4-1-2}$ (Brand et al., 1994) and UAS-GFP (Dickson, 1996), each crossed with one of the following Gal4 transgenes, da-GAL4 (Wodarz et al., 1995), GMR-GAL4 ${ }^{12}$ (Freeman, 1996), y w; actGAL4/CyO, $y^{+}$(Brand and Perrimon, 1993) and w; P[GawB $]^{(3) 31-1} / T M 6 C$ (Ito et al., 1997). 


\section{Drosophila media and culture}

Crosses were set up using four to five virgin females each of $U A S$-lacZ and $U A S-G F P$, mated to each of the drivers (da-GAL4, GMR-GAL4, act-GAL4/CyO, $y^{+}$and $\left.P[G a w B]^{(3) 31-1}\right)$ $T M 6 C$ ). All flies were maintained on standard cornmeal-yeast-agar media at $25^{\circ} \mathrm{C}$. Parental generation was transferred to new food after two days, and again two days after to increase the number of progeny. Critical class males were determined as those not presenting the balancer marker phenotypes of Tubby (TM6C), Stubble (TM6C), and Curly (CyO).

\section{Scanning electron microscopy}

Critical class male flies were isolated under $\mathrm{CO}_{2}$ and aged on standard cornmeal-yeastmolasses-agar media for three days, placed in $1.5-\mathrm{mL}$ microcentrifuge tubes and frozen at $-80^{\circ} \mathrm{C}$.

These animals were mounted on aluminum scanning electron microscope (SEM) studs using double-sided sticky tape, with left eye facing up for eye SEMs and ventral side down with legs removed and wings spread to the sides for dorsal notum SEMs. Progeny that inherit the transgenes $d a-G A L 4$ and $P[G a w B]^{l(3) 31-1}$ underwent dorsal notum analysis. The mounted flies were desiccated overnight and gold-coated using Electron Microscopy Science 500 Sputter Coater. Eyes were photographed at 150X magnification and dorsal nota were photographed at $80 \mathrm{X}$ magnification, using a Hitachi S-570 SEM.

Micrographs were analyzed using the ImageJ digital image analysis software (Abramoff et al., 2004). For the eye SEMs, the number of ommatidia was counted in each image $(\mathrm{N}=10$ per cross). The area $\left(\mu \mathrm{m}^{2}\right)$ of seven ommatidia (in a florette pattern) was determined for 10 sets, and the average area per ommatidium was calculated. Finally, overall eye area was measured. For dorsal notum analysis, the number of microchaetae on the dorsal notum was counted in each image ( $\mathrm{N}=25$ to 30 counts per genotype). The total area $\left(\mathrm{mm}^{2}\right)$ of the dorsal notum was also measured. Microchaete counts and total area were used to calculate the bristle density, expressed as number of microchaetae per $\mathrm{mm}^{2}$. All SEM data were exported into GraphPad Prism 5 and mean \pm standard error of means was plotted for each characteristic for each genotype. Statistical analyses were conducted using ANOVA, followed by Newman-Keuls post-tests to determine significance between pairs. Significance was considered at $\mathrm{P}<0.05$.

\section{Longevity assay}

Two hundred critical class males of each genotype were collected using gaseous carbon dioxide and kept on standard cornmeal-yeast-molasses-agar media at $25^{\circ} \mathrm{C}$. Flies were maintained in non-crowded conditions of 1 to 20 flies per vial. Flies were transferred to fresh media every two days (Staveley et al., 1990) and scored for the presence of dead adults. Survival data was transferred to the GraphPad Prism 5 program and were compared using ANOVA, followed by Newman-Keuls post-tests. Significance was considered to be $95 \%$, with $\mathrm{P}<0.05$.

\section{Time to eclosion (developmental assay)}

Virgin females $U A S$-lacZ and UAS-GFP and males of each driver line were collected and aged on standard cornmeal-yeast-molasses-agar media for three to four days before mating. 
Each cross was set up as described above in Drosophila culture, using three to four virgin females and two to three males. Flies were kept on the fresh food media for $6 \mathrm{~h}$ before being transferred to fresh media and kept another $6 \mathrm{~h}$. This was repeated four times, with several vials per cross.

The number of eclosed adults for each mating was recorded and the percentage eclosion calculated using:

$$
\% \text { eclosed }=\mathrm{n} / \mathrm{N} * 100 \%
$$

where $n$ is the number of eclosed adults for a cross on a given day, and $N$ is the total number of adults eclosed. Both male and female critical class flies were counted. Data was exported to and analyzed in GraphPad Prism 5.

\section{Climbing assay}

Approximately 200 critical class males of each cross were assayed for climbing ability according to a protocol developed in our laboratory (Todd and Staveley, 2004). Flies were assayed and maintained in groups of 10. Flies were kept on standard cornmeal-yeast-molasses-agar media at $25^{\circ} \mathrm{C}$ and assayed every seven days. Climbing ability was determined using a glass tube apparatus, $30 \mathrm{~cm}$ in length, $1.5 \mathrm{~cm}$ in diameter, marked with five $2-\mathrm{cm}$ sections with a buffer section at the top to limit interference between climbing flies. Climbing ability was scored after $10 \mathrm{~s}$ based on which of the five marked sections had been reached. Flies were scored 10 times per trial, and the climbing index calculated using:

$$
\text { Climbing index }=\Sigma(\mathrm{nm}) / \mathrm{N}
$$

where $n$ is the number of flies at a given section, $m$ is the section number (1-5) and $N$ is the total number of flies that climbed in that trial. Data were exported to GraphPad Prism 5 (GraphPad Software, Inc.) and each climbing index was subtracted from five and a non-linear regression curve was fit to each set of data. Slopes of curves were compared with $95 \%$ confidence intervals.

\section{RESULTS}

\section{Biometric analyses}

The development of ommatidia in the fly eye and microchaetae on the dorsal notum are two tightly controlled processes. Any interruption in these can produce phenotypic changes, which can be examined using biometric analysis. Scanning electron micrographs of the eyes and dorsal nota of flies expressing lac $Z$ and GFP in different patterns revealed alterations in development.

\section{Ubiquitous expression of GFP affects ommatidium size and microchaete density}

Examination of ubiquitous expression of $l a c Z$ and $G F P$ was conducted using the transgenes $d a-G A L 4$ and act-GAL4, which have been characterized to drive expression at low and high levels, respectively. Analyses of eye SEMs show that GFP expression does not greatly affect overall eye development. When directed in the pattern of $d a-G A L 4$, ommatidium number 
(747 \pm 4$)$ and eye area $\left(115,154 \pm 1714 \mu \mathrm{m}^{2}\right)$ were not unlike that seen for lacZ-expressing flies $\left(745 \pm 10 ; 114,234 \pm 2532 \mu \mathrm{m}^{2}\right)$ (Figure 1A). Ommatidium area was significantly larger in $U A S$ GFP; da-GAL4 flies $\left(216.6 \pm 2.62 \mu \mathrm{m}^{2}\right)$ compared to UAS-lacZ; da-GAL4 $\left(198.5 \pm 1.17 \mu \mathrm{m}^{2}\right)$ (Figure 1A, iv; Table 1). When directed by the act-GAL4 driver, lacZ and GFP expression did not affect eye area or ommatidium number or size (Figure 1B; Table 1). Ubiquitous expression of these two reporters had no significant effect on eye development. However, in the dorsal notum, low-level ubiquitous expression did affect microchaete density. The density of small bristles in $U A S-G F P$; da-GAL4 flies was significantly less $\left(583.6 \pm 10.9 \mathrm{~mm}^{2}\right)$ than UAS-lacZ; da-GAL4 flies $\left(667.1 \pm 7.96 \mathrm{~mm}^{2}\right)$ (Figure 2A; Table 2). The expression of $l a c Z$ and GFP genes had variable effects on the morphology of flies, as observed in the eye and dorsal notum.
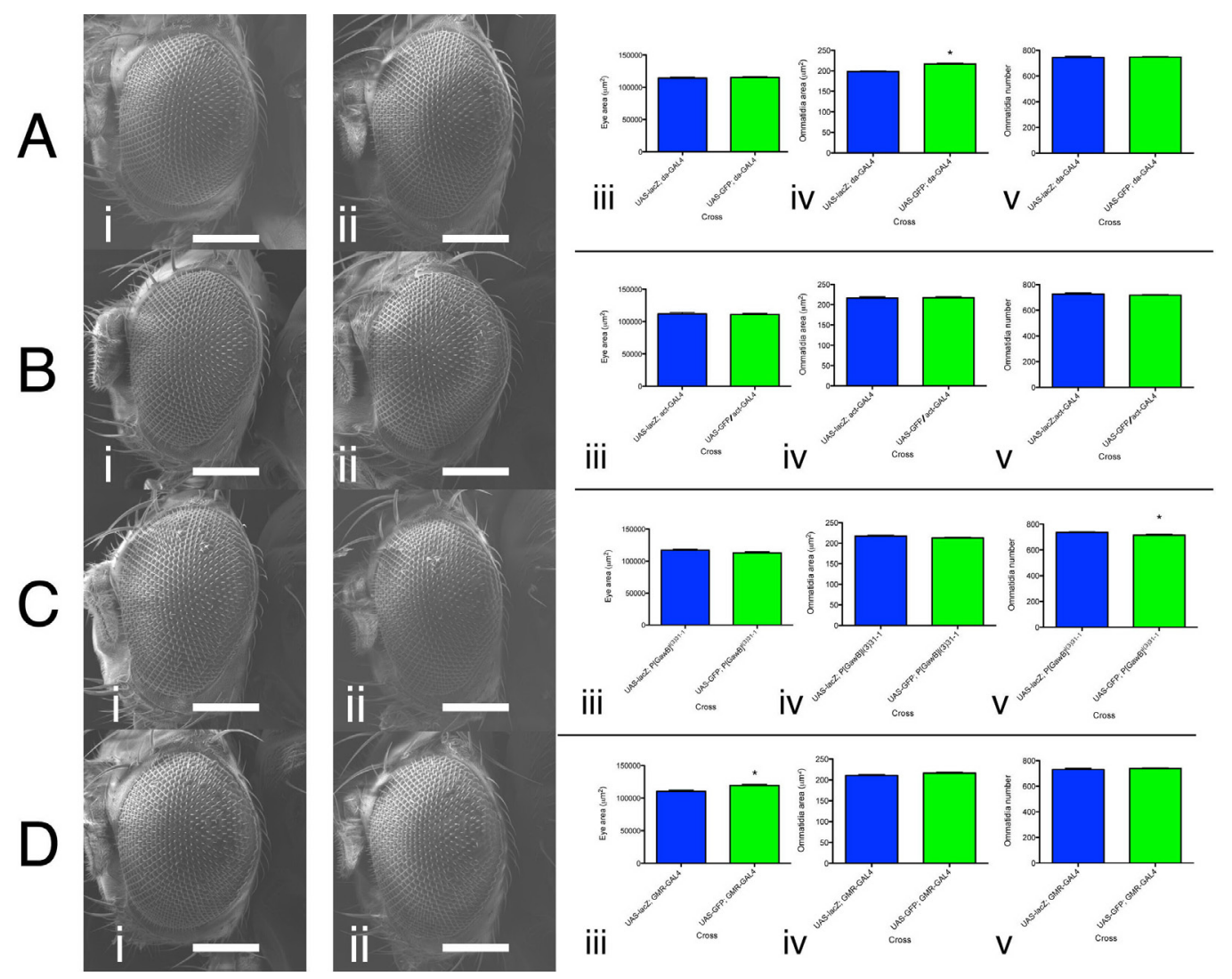

Figure 1. Biometric analyses of eye development influenced by GFP expression under the control of various transgenes. Scanning electron micrographs of left eyes from male flies. Scale bar (white) represents $160 \mu \mathrm{m}$. Genotypes are as follows: A. i $=U A S$-lacZ/da-GAL4; ii $=U A S-G F P / d a-G A L 4$. B. $\mathrm{i}=U A S$-lacZ/act-GAL4; ii $=U A S-G F P / a c t-G A L 4 ;$ C. $\mathrm{i}=U A S-l a c Z ; P[G a w B]^{(3) 31-1}$; ii $=U A S-G F P ; P[G a w B]^{(3) 31-1}$. D. i $=U A S-l a c Z / G M R-$ $G A L 4$; ii $=U A S-G F P / G M R-G A L 4$. Graphic representations of scanning electron micrograph eye analyses from $d a-$ GAL4 flies (A, iii-v), act-GAL4 flies (B, iii-v), P[GawB $]^{l(3) 31-1}$ flies (C, iii-v), and GMR-GAL4 flies (D, iii-v). UASlac $Z$ flies are presented in blue and $U A S-G F P$ flies are presented in green. Number of eyes analyzed from each cross $(\mathrm{N}=10)$. Graphic representations of eye area, ommatidium area and number are shown in iii, iv and v, respectively. Values are reported as means \pm SEM. *Represents significant difference calculated by Newman-Keuls post-tests. 
Table 1. Summary of eye scanning electron micrograph analyses: effect of GFP vs lacZ expression upon morphology.

\begin{tabular}{|c|c|c|c|}
\hline Genotype & Eye area $\left(\mu \mathrm{m}^{2}\right)$ & Ommatida area $\left(\mu \mathrm{m}^{2}\right)$ & Ommatida \# \\
\hline \multicolumn{4}{|l|}{ Low-level ubiquitous } \\
\hline$U A S-G F P ; d a-G A L 4$ & $115,154 \pm 1714$ & $217 \pm 3 \uparrow$ & $748 \pm 4$ \\
\hline$U A S-l a c Z ; d a-G A L 4$ & $111,966 \pm 2400$ & $198 \pm 1$ & $745 \pm 10$ \\
\hline \multicolumn{4}{|l|}{ High-level } \\
\hline$U A S-G F P ;$ act-GAL4 & $111,078 \pm 1716$ & $217 \pm 2$ & $739 \pm 5$ \\
\hline$U A S-l a c Z ;$ act-GAL4 & $111,873 \pm 2582$ & $216 \pm 3$ & $726 \pm 8$ \\
\hline \multicolumn{4}{|l|}{ Neurons and neuroblasts } \\
\hline$U A S-G F P ; P[G a w B]^{l(3) 31-1}$ & $113,069 \pm 1579$ & $213 \pm 2$ & $715 \pm 7 \downarrow$ \\
\hline$U A S-l a c Z ; P[G a w B]^{l(3) 31-1}$ & $117,111 \pm 1594$ & $217 \pm 2$ & $736 \pm 5$ \\
\hline \multicolumn{4}{|l|}{ Eye } \\
\hline$U A S-G F P ; G M R-G A L 4$ & $111,935 \pm 1854 \uparrow$ & $216 \pm 2$ & $718 \pm 4$ \\
\hline UAS-lacZ; GMR-GAL4 & $110,469 \pm 1723$ & $211 \pm 2$ & $731 \pm 10$ \\
\hline
\end{tabular}

Values are reported as means \pm SEM. Arrows indicate increase $(\uparrow)$ or decrease $(\downarrow)$ in treatment, and significance is denoted by the $\mathrm{P}$ value. Unless otherwise noted, there is no significant difference.

A

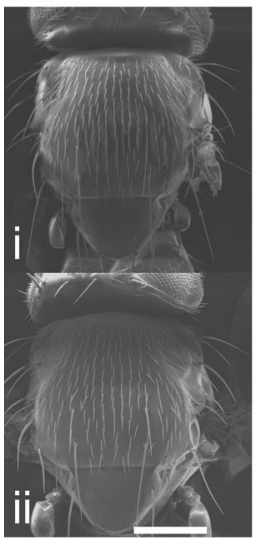

B

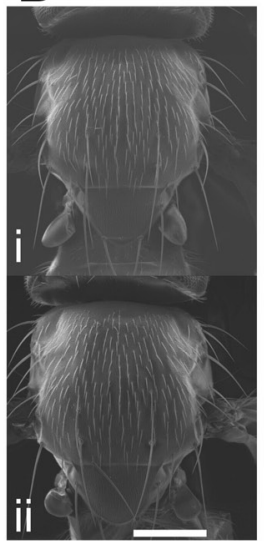

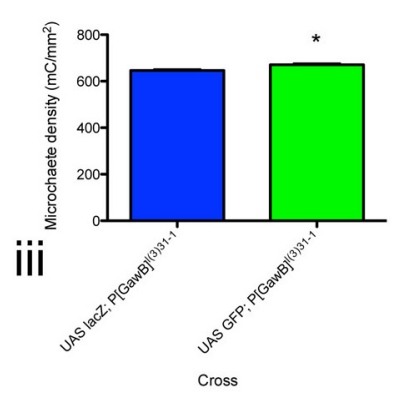

Figure 2. Biometric analysis of bristle density: GFP vs lacZ expression under the control of the ubiquitous transgene $d a-G A L 4$ and the neural transgene $P[G a w B]^{(3) 31-1}$. A. i-iii $=$ Ubiquitous $G F P$ expression $(\mathrm{N}=27)$ significantly decreases microchaete density compared to the expression of $\operatorname{lac} Z(\mathrm{~N}=29)$. B. $\mathrm{i}-\mathrm{iii}=$ Neural $G F P$ expression under $P[G a w B]^{(3) 31-1}$ $(\mathrm{N}=30)$ significantly increases microchaete density compared to lac $Z(\mathrm{~N}=29)$. UAS-lacZ flies are presented in blue and $U A S-G F P$ is presented in green. Scanning electron micrographs are shown in A (i and ii) and $\mathrm{B}$ (i and ii). Scale bar (white) represents $0.30 \mathrm{~mm}$. Graphic representation of microchaete density is shown in iii. Values are reported as means \pm SEM. *Represents significant difference by Newman-Keuls post-tests. Genotypes are as follows: A. $\mathrm{i}=U A S$-lacZ/ da-GAL4; ii $=U A S-G F P / d a-G A L 4$. B. $\mathrm{i}=U A S-l a c Z ; P[G a w B]^{(3) 31-1} ;$ ii $=U A S-G F P ; P[G a w B]^{l(3) 3 l-1}$.

Table 2. Summary of microchaete density on dorsal notum of flies with ubiquitous or neural GFP expression.

\begin{tabular}{lccc}
\hline Genotype & Microchaete density $\left(\mathrm{mC} / \mathrm{mm}^{2}\right)$ & P value (compared with UAS-lacZ cross) & Significant \\
\hline Low-level ubiquitous & $584 \pm 11$ & $<0.0001$ & Yes $\downarrow$ \\
$U A S-G F P ;$ da-GAL4 & $667 \pm 8$ & & \\
$U A S-l a c Z ;$ da-GAL4 & $671 \pm 6$ & $<0.0001$ & Yes $\uparrow$ \\
Neurons and neuroblasts & $646 \pm 5$ & & \\
$U A S-G F P ; P[G a w B]^{l(3) 31-1}$ & & & \\
$U A S-l a c Z ; P[G a w B]^{l(3) 3-1-1}$ & & & \\
\hline
\end{tabular}

Values are reported as means \pm SEM. Arrows indicate increase $(\uparrow)$ or decrease $(\downarrow)$ in treatment. 


\section{Neural GFP expression affects ommatidium number and microchaete density}

Analyses of neural expression of $l a c Z$ and $G F P$ were accomplished by using $P[G a w B]^{l(3) 3 l-1}$, which drives gene expression in the neurons and neuroblasts. Expression of $G F P$ via this driver did not greatly affect eye morphology compared to lacZ-expressing flies. The ommatidium number in UAS-GFP; $P[\text { GawB }]^{l(3) 31-l}$ flies $(714 \pm 7)$ was significantly less than in UAS-lacZ; $P[G a w B]^{l(3) 3 l-1}$ flies $(736 \pm 5)$ (Figure $1 \mathrm{C}$, v); however, ommatidium and eye areas were not different (Figure 1C, iii-iv). Microchaete density varied with neural expression of GFP compared to lacZ. Expression via the $P[G a w B]^{(3) 31-1}$ driver produced a higher microchaete density in $G F P$-expressing flies $\left(671.0 \pm 5.70 \mathrm{~mm}^{2}\right)$ versus lacZ-expressing flies $\left(646.5 \pm 5.18 \mathrm{~mm}^{2}\right)$ (Figure 2B; Table 2). Neural driver coupled with GFP expression altered some aspects of morphology compared to lac $Z$ expression.

\section{$G F P$ expression in the eye increases overall eye area}

Directed expression of lacZ and GFP was done using the GMR-GAL4 driver. This driver pushes gene expression posterior to the morphogenetic furrow, post-cell determination (Baker, 2001). GFP expression did not affect ommatidium number or size but did significantly increase overall eye area $\left(111,935 \pm 1854 \mathrm{~mm}^{2}\right)$ compared to lacZ $\left(110,469 \pm 1723 \mathrm{~mm}^{2}\right)$ (Figure 1D, Table 1). GFP expression seemed to affect eye development slightly when expression was driven in the eye, specifically.

\section{Behavioral analyses}

Behavioral analyses can show wide-ranging effects of gene expression. We performed a few assays that measured various aspects of development, including longevity, development time and climbing ability. We find that GFP expression significantly alters fly behavior compared to lac Z counterparts.

\section{Ubiquitous GFP expression affects longevity and climbing ability and can delay development time}

Ubiquitous expression of GFP was again completed using the transgenes $d a-G A L 4$ and act-GAL4, driving low and high expression, respectively. In both cases, longevity of $U A S$ GFP flies was decreased (Figure $3 \mathrm{~A}$ and B). The median life span of flies from these crosses was 58 days, 10-12 days shorter than lacZ flies, and the maximum life span varied from 78 (UAS-GF; da-GAL4) to 82 days (UAS-GFP/act-GAL4) (Table 3). Although flies with the $d a-$ GAL4 driver eclosed at the same time as the control (Figure 4A), development time differed significantly when GFP was expressed with the act-GAL4 driver (Figure 4B). About $50 \%$ of flies from this cross eclosed one day earlier than the lac Z flies (at 10 days versus 11). Climbing tests were conducted over several weeks and analyses were stopped when $75 \%$ of the initial number of flies had died. Expression of UAS-GFP using act-GAL4 decreases climbing ability over time. The climbing indices for UAS-GFP; da-GAL4 flies remain similar to UAS-lacZ; $d a-G A L 4$ flies (Figure 5A and B). The climbing ability of UAS-GFP/act-GAL4 flies, however, was significantly lower than that of the lacZ control (Figure 5B). Overall, GFP expression 
throughout the organism had variable effects on eclosion time and climbing ability, and severely decreased longevity.
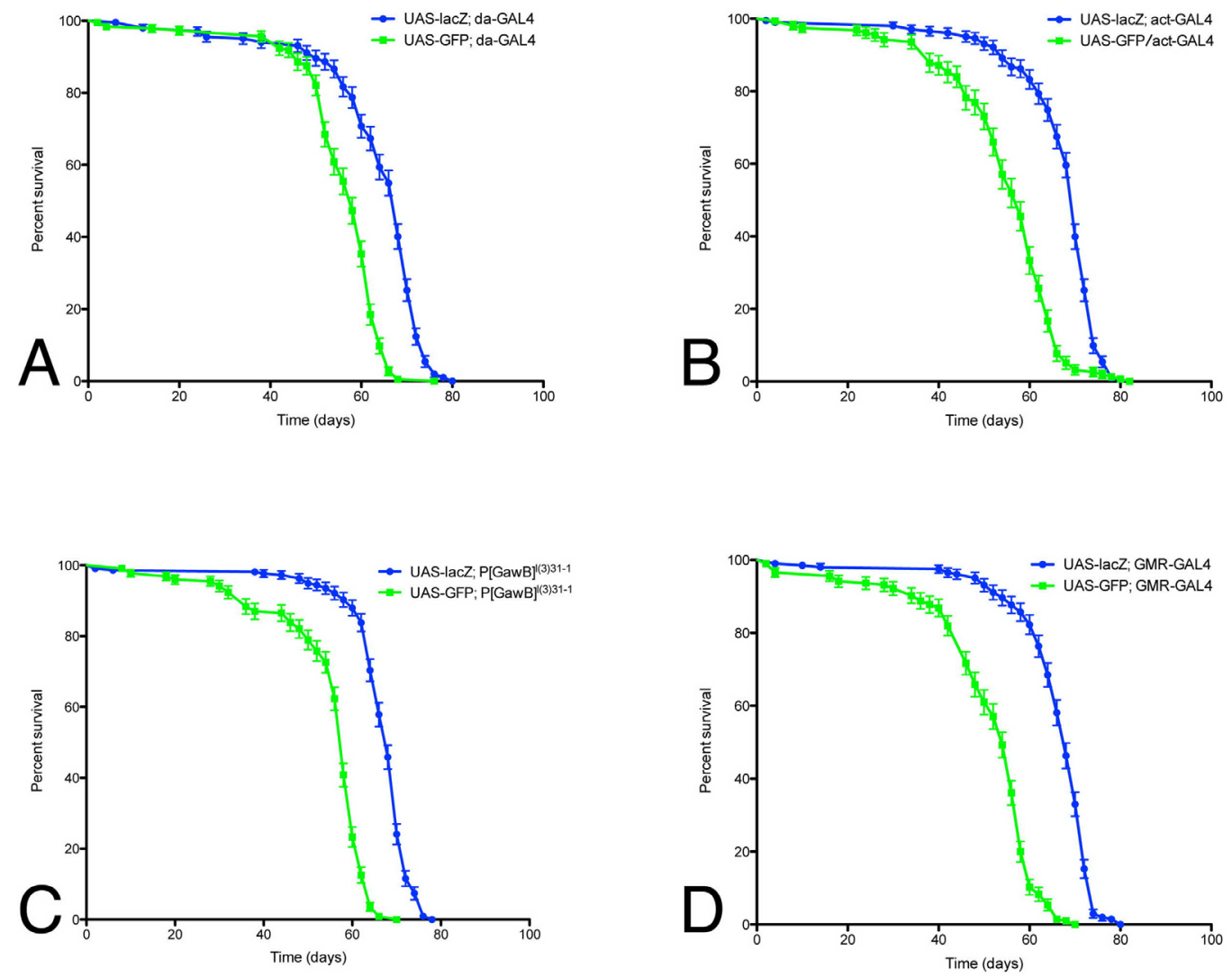

Figure 3. Behavioral analysis: effect of GFP expression throughout the organism, in the neurons and in the eye upon longevity. GFP expression significantly decreases lifespan in flies. Graphic representations of longevity for ubiquitous expression: A. da-GAL4 and B. act-GAL4 drivers; neural expression: C. $P[G a w B]^{(3) 31-1}$, and eye expression: D. GMRGAL4. Longevity is shown as percent survival $(\mathrm{P}<0.05$, determined by log-rank). Error bars $=\mathrm{SEM}$.

\begin{tabular}{|c|c|c|c|c|c|}
\hline Genotype & $\begin{array}{l}\text { Initial number of } \\
\text { flies analyzed }(\mathrm{N})\end{array}$ & $\begin{array}{l}\text { Median survival } \\
\text { day }(50 \%)\end{array}$ & $\begin{array}{c}\text { Maximum lifespan } \\
\text { (day) }\end{array}$ & $\begin{array}{c}\mathrm{P} \text { value (compared with } \\
\text { UAS-lac } Z \text { cross) }\end{array}$ & Significant \\
\hline \multicolumn{6}{|l|}{ Low-level } \\
\hline$U A S-G F P ; d a-G A L 4$ & 184 & 58 & 78 & \multirow[t]{2}{*}{$<0.0001$} & \multirow{2}{*}{ Yes $\downarrow$} \\
\hline UAS-lacZ; da-GAL4 & 202 & 68 & 80 & & \\
\hline \multicolumn{6}{|l|}{ High-level } \\
\hline UAS-GFP; act-GAL4 & 156 & 58 & 82 & \multirow[t]{2}{*}{$<0.0001$} & \multirow[t]{2}{*}{ Yes $\downarrow$} \\
\hline UAS-lacZ; act-GAL4 & 203 & 70 & 82 & & \\
\hline \multicolumn{6}{|l|}{ Neurons and neuroblasts } \\
\hline$U A S-G F P ; P[\operatorname{GawB}]^{(3) 331-1}$ & 223 & 58 & 70 & \multirow{2}{*}{$<0.0001$} & \multirow[t]{2}{*}{ Yes $\downarrow$} \\
\hline UAS-lacZ; P[GawB] $]^{(3) 31-1}$ & 216 & 68 & 78 & & \\
\hline \multicolumn{6}{|l|}{ Eye } \\
\hline$U A S-G F P ; G M R-G A L 4$ & 205 & 54 & 70 & \multirow[t]{2}{*}{$<0.0001$} & \multirow[t]{2}{*}{ Yes $\downarrow$} \\
\hline UAS-lacZ; GMR-GAL4 & 203 & 68 & 80 & & \\
\hline
\end{tabular}

Survival curves analyzed using the log-rank test. Arrows indicate decrease $(\downarrow)$ in treatment. 

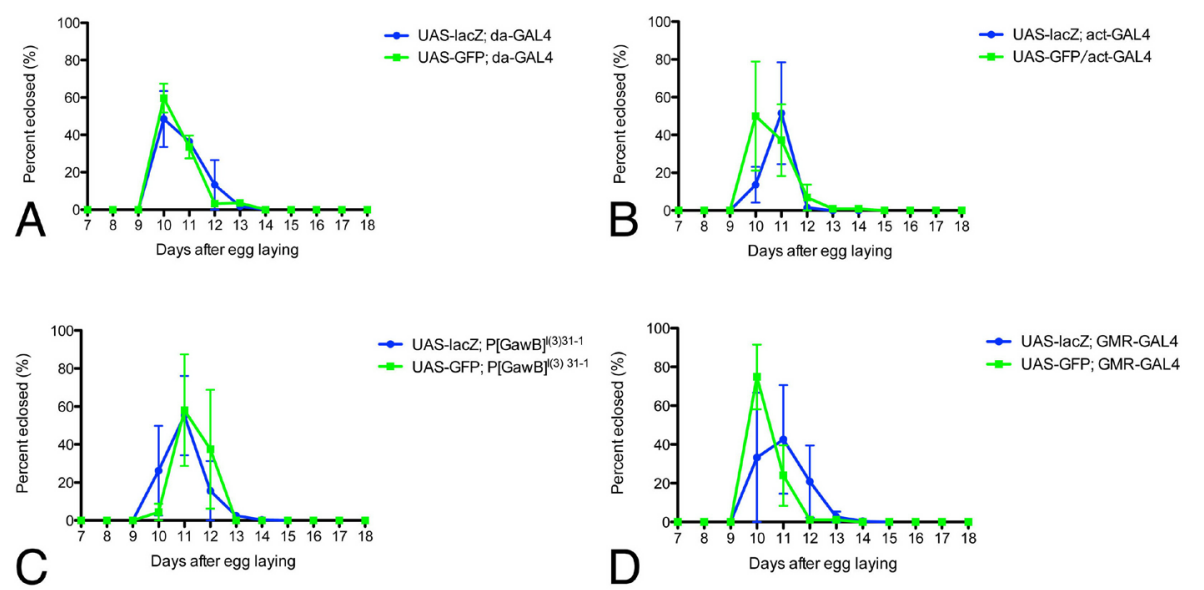

Figure 4. Behavioral analysis: effect of GFP expression throughout the organism, in the neurons and in the eye upon development (eclosion) time. GFP expression significantly does not have a significant effect on eclosion time in flies. Graphic representations of eclosion time (development time) for ubiquitous expression: A. da-GAL4 and B. act-GAL4 drivers; neural expression: C. $P[G a w B]^{(3) 31-1}$, and eye expression: D. GMR-GAL4. Percentage of total flies eclosed each day is shown. Error bars $=$ SEM.
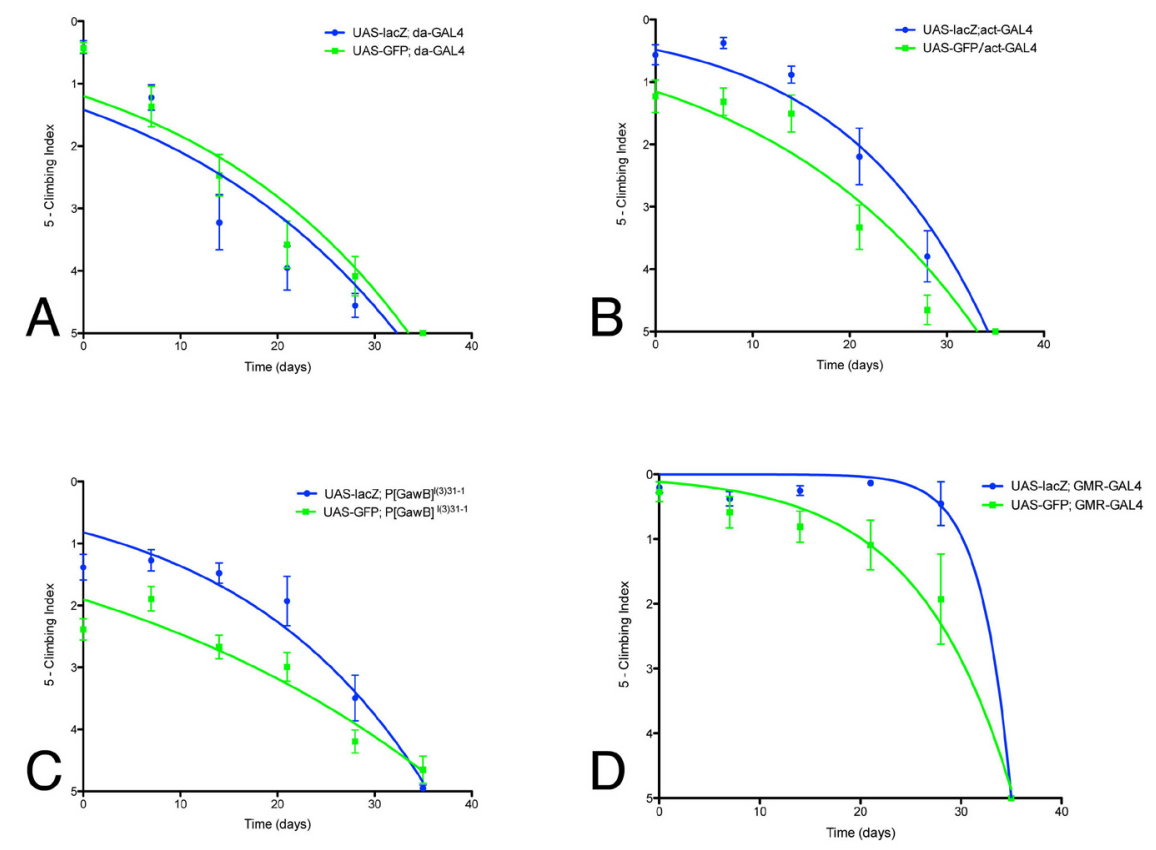

Figure 5. Behavioral analysis: effect of GFP expression throughout the organism, in the neurons and in the eye upon climbing ability. GFP expression produces flies with significantly decreased climbing ability when driven by the ubiquitous transgene act-GAL4, the neural transgene $P[G a w B]^{(3) 31-1}$ and eye-specific transgene GMR-GAL4. Graphic representations of longevity for ubiquitous expression: A. da-GAL4 and B. act-GAL4 drivers; neural expression: C. $P[G a w B]^{(3) 31-1}$, and eye expression: D. GMR-GAL4. Climbing ability was determined via non-linear curve fit $(\mathrm{CI}=95 \%)$. Error bars $=$ SEM. 


\section{Expression of $G F P$ in the brain and neurons affects longevity and climbing ability}

We found that neural GFP expression can affect longevity and locomotor ability in flies. Neural expression was achieved using the $P[G a w B]^{(3) 31-1}$ driver. Longevity studies of these flies showed significantly decreased life span in GFP-expressing flies (Figure 3C). The median life span was 58 days for UAS-GFP; $[\text { [GawB }]^{l(3) 3 l-1}$ flies compared to UAS-lacZ; $P[G a w B]^{l(3) 3 l-1}$ flies with 68 days (Table 3). Maximum life spans for GFP and lacZ crosses did not differ significantly. Neural GFP expression did not affect eclosion time as GFP flies eclosed on the same day as lacZ flies - 12 days (Figure 4C). Expression of GFP produced flies with poor climbing ability when expressed in the neurons and neuroblasts (Figure 5C). Clearly, the directed neural expression of GFP affected the behavior of flies when compared to controls expressing UAS-lacZ.

\section{GFP expression in the eye affects longevity, development time and climbing ability}

Directed GFP expression in the eye was accomplished using the GMR-GAL4 driver. Longevity of $U A S-G F P ; G M R-G A L 4$ flies was significantly decreased compared to the control (Figure 3D). The median life span was shorter, 54 days compared to UAS-lacZ; GMR-GAL4 with 68 days, and the maximum life span 70 to 80 days (Table 3). Development time of UASGFP; GMR-GAL4 flies was shorter by one day (10 days) compared to the control (11 days) (Figure 4D). GFP expression in the eye also produced poor climbing ability (Figure 5D). GFP expression in the eye significantly affected behavior in these fruit flies.

\section{DISCUSSION}

The GFP gene is commonly used as a marker that allows visualization of tissues and biological events during development. The use of GFP has expanded into other species, including mice and zebrafish (Nowotschin et al., 2009; Zhan and Gong, 2010). Siegmund and Korge (2001) used GFP expression to visualize the ring gland in the Drosophila brain. UAS$G F P$-bearing flies have been used in various assays, as it is believed that GFP expression has no physiological effect in flies. We demonstrated that this is not always the case. While GFP expression is not shown to affect physical characteristics significantly, such as in the eye and dorsal notum, GFP expression can affect physiological phenotypes.

Scanning electron micrograph analyses of the eye did not reveal many significant differences when compared to the lacZ-expressing flies. Slight variations in eye area, ommatidium area or count were observed with some of the trangenes. However, the nature of these differences seems minor, suggesting there is no serious effect of GFP expression on development or neurogenesis in the eye. Examination of bristle density can be a good indicator of neurogenesis. A recent study, which investigated the role of Hip1 in neurogenesis (Moores et al., 2008), found that analysis of microchaete density is a sensitive assay that can assess subtle changes to cell signaling. Here we found that GFP expression under the influence of $d a-G A L 4$ and $P[G a w B]^{l(3) 31-1}$ transgenes can affect bristle density, indicating that GFP may influence the cell signaling patterns important in bristle development and neurogenesis. These SEM data indicate that use of GFP in assays with certain transgenes may need to be carefully evaluated to ensure that GFP expression has not affected events during development, producing altered phenotypes. 
Expression of GFP may only slightly affect neuronal development and eye and dorsal notum morphology. However, its expression significantly decreases longevity and climbing ability when directed in neural tissues and throughout the organism. The influence upon longevity may greatly influence continual developmental experiments, by introducing bias. Expression of GFP affects the condition of the flies over the long-term, resulting in poor climbing ability. This suggests that analyses of long-term locomotor assays (reviewed by Iliadi and Boulianne, 2010) or muscle development in flies may be affected when using GFP as a marker (Soler et al., 2004). If GFP influences fly health enough over time to not only decrease longevity but also locomotor (climbing) ability, its use in long-term development assays may be problematic, when expression is directed with the drivers that we used here.

Perhaps controlling the initiation of GFP expression, as with heat-shock promoters, would reduce the possible effects on development, allowing analysis in longitudinal assays (Grover et al., 2008). Caution should be taken when GFP is used in long-term experiments with any drivers.

\section{ACKNOWLEDGMENTS}

This research was funded by Memorial University of Newfoundland School of Graduate Studies Fellowship to R.M.S. Mawhinney and by a National Sciences and Engineering Council of Canada Discovery grant to B.E. Staveley. Thanks to Liqiu Men and Gary Collins for their technical assistance. Special thanks to Jennifer Slade, Amy Todd, Kate Bassett, Renee Mulrooney, Colleen Connors, Katie Antle, and Kim Chafe for helpful discussion and technical assistance with experimental analysis.

\section{REFERENCES}

Abramoff MD, Magalhães PJ and Ram SJ (2004). Image processing with ImageJ. Biophotonics Int. 11: 36-42.

Baker NE (2001). Cell proliferation, survival, and death in the Drosophila eye. Semin. Cell Dev. Biol. 12: 499-507.

Bier E, Vaessin H, Shepherd S, Lee K, et al. (1989). Searching for pattern and mutation in the Drosophila genome with a P-lacZ vector. Genes Dev. 3: 1273-1287.

Brand AH and Perrimon N (1993). Targeted gene expression as a means of altering cell fates and generating dominant phenotypes. Development 118: 401-415.

Brand AH, Manoukian AS and Perrimon N (1994). Ectopic expression in Drosophila. Methods Cell Biol. 44: 635-654.

Chalfie M, Tu Y, Euskirchen G, Ward WW, et al. (1994). Green fluorescent protein as a marker for gene expression. Science 263: 802-805.

Dickson B (1996). Transgenic lines 1010T2 and 1010T10. Personal Communication to FlyBase. Available at [http:// flybase.org/reports/FBrf0086268.html]. Accessed August 10, 2010.

Duffy JB (2002). GAL4 system in Drosophila: a fly geneticist's Swiss army knife. Genesis 34: 1-15.

Freeman M (1996). Reiterative use of the EGF receptor triggers differentiation of all cell types in the Drosophila eye. Cell 87: 651-660.

Grover D, Yang J, Tavare S and Tower J (2008). Simultaneous tracking of fly movement and gene expression using GFP. BMC Biotechnol. 8: 93.

Iliadi KG and Boulianne GL (2010). Age-related behavioral changes in Drosophila. Ann. N. Y. Acad. Sci. 1197: 9-18.

Ito K, Awano W, Suzuki K, Hiromi Y, et al. (1997). The Drosophila mushroom body is a quadruple structure of clonal units each of which contains a virtually identical set of neurones and glial cells. Development 124: 761-771.

Kramer JM and Staveley BE (2003). GAL4 causes developmental defects and apoptosis when expressed in the developing eye of Drosophila melanogaster. Genet. Mol. Res. 2: 43-47.

Manseau L, Baradaran A, Brower D, Budhu A, et al. (1997). GAL4 enhancer traps expressed in the embryo, larval brain, imaginal discs, and ovary of Drosophila. Dev. Dyn. 209: 310-322.

Misteli T and Spector DL (1997). Applications of the green fluorescent protein in cell biology and biotechnology. Nat. 
Biotechnol. 15: 961-964.

Moores JN, Roy S, Nicholson DW and Staveley BE (2008). Huntingtin interacting protein 1 can regulate neurogenesis in Drosophila. Eur. J. Neurosci. 28: 599-609.

Nowotschin S, Eakin GS and Hadjantonakis AK (2009). Live-imaging fluorescent proteins in mouse embryos: multidimensional, multi-spectral perspectives. Trends Biotechnol. 27: 266-276.

O'Kane CJ and Gehring WJ (1987). Detection in situ of genomic regulatory elements in Drosophila. Proc. Natl. Acad. Sci. U. S. A. 84: 9123-9127.

Phelps CB and Brand AH (1998). Ectopic gene expression in Drosophila using GAL4 system. Methods 14: 367-379.

Rosochacki SJ and Matejczyk M (2002). Green fluorescent protein as a molecular marker in microbiology. Acta Microbiol. Pol. 51: 205-216.

Siegmund T and Korge G (2001). Innervation of the ring gland of Drosophila melanogaster. J. Comp. Neurol. 431: 481-491.

Soler C, Daczewska M, Da Ponte JP, Dastugue B, et al. (2004). Coordinated development of muscles and tendons of the Drosophila leg. Development 131: 6041-6051.

Staveley BE, Phillips JP and Hilliker AJ (1990). Phenotypic consequences of copper-zinc superoxide dismutase overexpression in Drosophila melanogaster. Genome 33: 867-872.

Todd AM and Staveley BE (2004). Novel assay and analysis for measuring climbing ability in Drosophila. Dros. Inf. Serv. 87: 101-107.

Wang S and Hazelrigg T (1994). Implications for bcd mRNA localization from spatial distribution of exu protein in Drosophila oogenesis. Nature 369: 400-403.

Wodarz A, Hinz U, Engelbert M and Knust E (1995). Expression of crumbs confers apical character on plasma membrane domains of ectodermal epithelia of Drosophila. Cell 82: 67-76.

Yeh E, Gustafson K and Boulianne GL (1995). Green fluorescent protein as a vital marker and reporter of gene expression in Drosophila. Proc. Natl. Acad. Sci. U. S. A. 92: 7036-7040.

Zhan H and Gong Z (2010). Delayed and restricted expression of UAS-regulated GFP gene in early transgenic zebrafish embryos by using the GAL4/UAS system. Mar. Biotechnol. 12: 1-7. 